\title{
STATIONARY MEASURES AND PHASE TRANSITION FOR A CLASS OF PROBABILISTIC CELLULAR AUTOMATA
}

\author{
Paolo Dai Pra ${ }^{1}$, Pierre-Yves Louis ${ }^{2}$ and Sylvie Relly ${ }^{3,4}$
}

\begin{abstract}
We discuss various properties of Probabilistic Cellular Automata, such as the structure of the set of stationary measures and multiplicity of stationary measures (or phase transition) for reversible models.
\end{abstract}

Mathematics Subject Classification. 60G60, 60J10, 60K35, 82C20, 82B26.

Received December 4, 2001. Revised March 11, 2002.

\section{INTRODUCTION}

Probabilistic Cellular Automata (PCA) are discrete-time Markov chains on a product space $S^{\Lambda}$ (configuration space) whose transition probability is a product measure. In this paper, $S$ is assumed to be a finite set (spin space), and $\Lambda$ (set of sites) a subset, finite or infinite, of $\mathbb{Z}^{d}$. The fact that the transition probability $P\left(\mathrm{~d} \sigma \mid \sigma^{\prime}\right)$, $\sigma, \sigma^{\prime} \in S^{\Lambda}$, is a product measure means that all spins $\left\{\sigma_{i}: i \in \Lambda\right\}$ are simultaneously and independently updated (parallel updating). This transition mechanism differs from the one in the most common Gibbs samplers (e.g. $[3,8]$ ), where only one site is updated at each time step (sequential updating).

Several properties of PCA's, mainly of general and qualitative nature, have been investigated $[5,7,14,18,23]$. As far as we know, however, sharper properties like e.g. rate of convergence to equilibrium or use of parallel dynamics in perfect sampling, have not yet been investigated. PCA's are hard to analyze mainly for the following reason. Suppose $\Lambda$ is a finite subset of $\mathbb{Z}^{d}$, and let $\mu$ be a given probability on $S^{\Lambda}$. To fix ideas, we may think of $\mu$ as a finite volume Gibbs measure for a given interaction and assigned boundary conditions. It is simple to construct Markov chains on $S^{\Lambda}$ with sequential updating which have $\mu$ as reversible measure. Transition probabilities are given in simple form in terms of $\mu$, and reversibility immediately implies that $\mu$ is an stationary measure for the dynamics. Quite differently, for a given $\mu$, there is no general recipe to construct a PCA for which $\mu$ is stationary. In particular, there exists Gibbs measures on $S^{\mathbb{Z}^{2}}$ such that no PCA admits them as stationary reversible measures (cf. Th. 4.2 in [5] and Rem. 3.1).

Despite of this discouraging starting point, other aspects of PCA's make them interesting stochastic models, and motivate further investigation.

\footnotetext{
Keywords and phrases: Probabilistic Cellular Automata, stationary measure, Gibbs measure.

1 Dipartimento di Matematica Pura e Applicata, Università di Padova, Via Belzoni 7, 35131 Padova, Italy;

e-mail: daipra@math.unipd.it

2 Laboratoire de Statistique et Probabilités, FRE 2222 du CNRS, UFR de Mathématiques, Université Lille 1,

59655 Villeneuve-d'Ascq Cedex, France; e-mail: louis@lps.univ-lille1.fr

${ }^{3}$ Weierstraß-Institut für Angewandte Analysis und Stochastik, Mohrenstraße 39, 10117 Berlin, Germany;

e-mail: roelly@wias-berlin.de

4 Permanent address: CMAP, UMR 7641 du CNRS, École Polytechnique, 91128 Palaiseau Cedex, France.
} 
1. For simulation and sampling, PCA's are natural stochastic algorithms for parallel computing. At least in some simple models (see Sect. 3) it is interesting to evaluate their performance versus algorithms with sequential updating. This will be the subject of a forthcoming paper.

2. In opposition to dynamics with sequential updating, it is simple to define PCA's in infinite volume without passing to continuous time. One may try to study, for instance, convergence to equilibrium in infinite volume, or in finite volume uniformly in the volume size. Although some perturbative methods are available (see [20], Chap. 7, Refs. [17, 18]), a theory corresponding the one in [21] in continuous time, is yet to be developed.

3. PCA's that are reversible with respect to a Gibbs measure $\mu$ have been completely characterized in [11]. In particular it has been shown that only a small class of Gibbs measures may be reversible for a PCA. For such PCA's one can investigate metastable behavior. A first step in this direction is done in [2].

The present paper is a small step toward a better understanding of PCA's. Our objective is first to present some links between the sets of reversible, resp. stationary, resp. Gibbs measures for general PCA's. We then illustrate these results on a particular class of reversible PCA's already introduced in [2].

More precisely it was proved in [11] that for PCA's possessing a reversible Gibbs measure w.r.t. a potential $\Phi$, all reversible measures are gibbsian w.r.t the same potential. We prove a similar statement on the set of stationary measures: for a general PCA, if one shift invariant stationary measure is Gibbsian for a potential $\Phi$, then all shift invariant stationary measures are Gibbsian w.r.t. the same potential $\Phi$ (see Prop. 2.2). This induces that for a class of local, shift invariant, non-degenerated, reversible PCA the reversible measures coincide with the Gibbsian stationary ones (Rem. 3.2).

Applying this general statements to the class of PCA's considered in [2], one can do explicit a stationary measure which is in fact Gibbsian w.r.t. a certain potential $\Phi$ we write down (cf. Prop. 3.2); we show that, for sufficiently small values of the temperature parameter, phase transition occurs that is there are several Gibbs measures w.r.t. $\Phi$. At least in certain cases, existence of phase transition would follow from general expansion arguments, like Pirogov-Sinai theory. We have preferred here, however, to use "softer" contour arguments. The understanding of the right notion of contour for a specific model is in any case useful in many respects (percolation, block dynamics, ...).

However, unlike what happens with sequential updating, not all these Gibbs measures need to be stationary for the infinite volume PCA, the non-stationary ones being periodic with period two. To conclude, we exhibit a Gibbs measure which is not stationary for the associated PCA.

\section{Shift inVARIANt PRobabilistic CELlular AUtomata}

Let $S$ be a finite set. For $\sigma \in S^{\mathbb{Z}^{d}}, \sigma=\left(\sigma_{i}\right)_{i \in \mathbb{Z}^{d}}$, and $\Lambda \subset \mathbb{Z}^{d}$, we let $\sigma_{\Lambda} \in S^{\Lambda}$ its restriction to $\Lambda$. Sometimes, when no confusion arises, we omit the index $\Lambda$ in $\sigma_{\Lambda}$.

A time-homogeneous Markov chain on $S^{\Lambda}$ is determined, in law, by its transition probabilities $P_{\Lambda}(\mathrm{d} \sigma \mid \eta)$. If $P_{\Lambda}(\mathrm{d} \sigma \mid \eta)$ is a product measure, as a probability measure on $S^{\Lambda}$, then we say that the Markov chain is a Probabilistic Cellular Automaton. More explicitly

$$
P_{\Lambda}(\mathrm{d} \sigma \mid \eta)=\otimes_{i \in \Lambda} P_{i}\left(\mathrm{~d} \sigma_{i} \mid \eta\right)
$$

and

$$
P_{i}\left(\sigma_{i}=s \mid \eta\right) \equiv p_{i}(s \mid \eta), \quad s \in S
$$

In the case $\Lambda=\mathbb{Z}^{d}$, we omit the index $\Lambda$ in $P_{\Lambda}(\mathrm{d} \sigma \mid \eta)$. In this case, we say that a PCA is shift invariant if, for every $i \in \mathbb{Z}^{d}, s \in S, \eta \in S^{\mathbb{Z}^{d}}$, we have

$$
p_{i}(s \mid \eta)=p_{0}\left(s \mid \theta_{i} \eta\right)
$$


where $\theta_{i}$ is the shift in $\mathbb{Z}^{d}:\left(\theta_{i} \eta\right)_{j}=\eta_{i+j}$ for every $j \in \mathbb{Z}^{d}$. A shift invariant PCA is said to be local if, for each $s \in S$, the map $\eta \rightarrow p_{0}(s \mid \eta)$ is local, i.e. it depends on a finite number of components of $\eta$.

From now on, all PCA's we consider in this paper satisfy the non degeneration condition:

$$
p_{0}(s \mid \eta)>0, \quad \forall s \in S, \eta \in S^{\mathbb{Z}^{d}}
$$

This means that we are dealing with dynamics which can not contain a deterministic component.

In this paper we are mostly interested in stationary measures for PCA's. For this purpose we recall the notion of Gibbs measure on $S^{\mathbb{Z}^{d}}$. A shift invariant potential $\Phi$ is a family $\left\{\Phi_{\Lambda}: \Lambda \subset \mathbb{Z}^{d},|\Lambda|<+\infty\right\}$ of maps $\Phi_{\Lambda}: S^{\Lambda} \rightarrow \mathbb{R}$ with the properties

i. for all $i \in \mathbb{Z}^{d}, \Lambda \subset \mathbb{Z}^{d}$ finite:

$$
\Phi_{\Lambda+i}=\Phi_{\Lambda} \circ \theta_{i}
$$

ii.

$$
\sum_{\Lambda \ni 0}\left\|\Phi_{\Lambda}\right\|_{\infty}<+\infty
$$

Here and later $|\Lambda|$ denotes the cardinality of $\Lambda$. Letting $H_{\Lambda}(\sigma)=\sum_{A \cap \Lambda \neq \emptyset} \Phi_{A}(\sigma)$ and choosing $\tau \in S^{\mathbb{Z}^{d}}$, also write $H_{\Lambda}^{\tau}\left(\sigma_{\Lambda}\right)=H_{\Lambda}\left(\sigma_{\Lambda} \tau_{\Lambda^{c}}\right)$, where $\sigma_{\Lambda} \tau_{\Lambda^{c}}$ is the element of $S^{\mathbb{Z}^{d}}$ which coincides with $\sigma$ on $\Lambda$ and with $\tau$ on $\Lambda^{c}$. The finite volume Gibbs measure on $S^{\Lambda}$ with boundary condition $\tau$ is given by

$$
\mu_{\Lambda}^{\tau}\left(\sigma_{\Lambda}\right)=\frac{\exp \left[-H_{\Lambda}^{\tau}\left(\sigma_{\Lambda}\right)\right]}{Z_{\Lambda}^{\tau}},
$$

where $Z_{\Lambda}^{\tau}$ is the normalization factor. A probability measure $\mu$ on $S^{\mathbb{Z}^{d}}$ is said to be Gibbsian for the potential $\Phi$, and we write $\mu \in \mathcal{G}(\Phi)$ if for every $\Lambda \subset \mathbb{Z}^{d}$ finite and $\sigma \in S^{\mathbb{Z}^{d}}$

$$
\mu\left(\left\{\eta: \eta_{\Lambda}=\sigma_{\Lambda}\right\} \mid \eta_{\Lambda^{c}}=\tau_{\Lambda^{c}}\right)=\mu_{\Lambda}^{\tau}\left(\sigma_{\Lambda}\right)
$$

for $\mu$-a.e. $\tau$. If $\mu$ is shift-invariant, i.e. $\mu \circ \theta_{i}=\mu$ for all $i \in \mathbb{Z}^{d}$, then we write $\mu \in \mathcal{G}_{s}(\Phi)$. More generally, we let $\mathcal{P}$ (resp. $\mathcal{P}_{s}$ ) be the set of probability measures (resp. shift-invariant probability measures) on $S^{\mathbb{Z}^{d}}$.

Given $\Lambda \subset \mathbb{Z}^{d}$, we denote by $\mathcal{F}_{\Lambda}$ the $\sigma$-field on $S^{\mathbb{Z}^{d}}$ generated by the projection $\sigma \rightarrow \sigma_{\Lambda}$. For $\nu \in \mathcal{P}, \pi_{\Lambda} \nu$ is the restriction of $\nu$ to $\mathcal{F}_{\Lambda}$. We will use, for $\nu, \mu \in \mathcal{P}$, the notion of local relative entropy:

$$
h_{\Lambda}(\nu \mid \mu)=\sum_{\sigma_{\Lambda}} \pi_{\Lambda} \nu\left(\sigma_{\Lambda}\right) \log \frac{\pi_{\Lambda} \nu\left(\sigma_{\Lambda}\right)}{\pi_{\Lambda} \mu\left(\sigma_{\Lambda}\right)}
$$

with $\Lambda \subset \mathbb{Z}^{d}$ finite, and of specific relative entropy

$$
h(\nu \mid \mu)=\limsup _{\Lambda \uparrow \mathbb{Z}^{d}} \frac{1}{|\Lambda|} h_{\Lambda}(\nu \mid \mu)
$$

where in the limit above $\Lambda$ varies over hypercubes centered in the origin. It is easily seen that $0 \leq h(\nu \mid \mu) \leq+\infty$. In the case of $\mu \in \mathcal{G}_{s}(\Phi)$ for a potential $\Phi$, in $(2.2) \pi_{\Lambda} \mu\left(\sigma_{\Lambda}\right)$ can be replaced by $\mu_{\Lambda}^{\tau}\left(\sigma_{\Lambda}\right)$, for an arbitrary $\tau$, without changing the limit in (2.3). Moreover, for $\mu \in \mathcal{G}_{s}(\Phi)$ and $\nu \in \mathcal{P}_{s}$, the limsup in (2.3) is actually a limit. In this case the Gibbs variational principle states that, for $\nu \in \mathcal{P}_{s}, h(\nu \mid \mu)=0$ if and only if $\nu \in \mathcal{G}_{s}(\Phi)$; so $h(\nu \mid \mu)$ represents a notion of (pseudo-) distance of $\nu$ from $\mathcal{G}_{s}(\Phi)$. 
We now define a corresponding notion of specific relative entropy for transition probabilities, that will be used to measure distance between two dynamics. Let $P(\mathrm{~d} \sigma \mid \eta)$ and $Q(\mathrm{~d} \sigma \mid \eta)$ two transition probabilities on $S^{\mathbb{Z}^{d}}$, and $\nu \in \mathcal{P}$. We define

$$
\mathcal{H}_{\nu}(P \mid Q)=\limsup _{\Lambda \uparrow \mathbb{Z}^{d}} \frac{1}{|\Lambda|} \int h_{\Lambda}(P(\cdot \mid \eta) \mid Q(\cdot \mid \eta)) \nu(\mathrm{d} \eta)
$$

Clearly $\mathcal{H}_{\nu}(P \mid Q) \geq 0$. By conditioning to $\sigma$ the joint law $Q_{\nu}(\mathrm{d} \sigma, \mathrm{d} \eta) \equiv P(\mathrm{~d} \sigma \mid \eta) \nu(\mathrm{d} \eta)$ we obtain the backward transition probability, that we denote by $\hat{P}_{\nu}(\mathrm{d} \eta \mid \sigma)$. We also let $P \nu(\mathrm{d} \sigma)$ be given by

$$
P \nu(A)=\int P(A \mid \eta) \nu(\mathrm{d} \eta)
$$

for $A \subset S^{\mathbb{Z}^{d}}$ measurable. If $P \nu=\nu$ we say that $\nu$ is stationary for $P(\mathrm{~d} \sigma \mid \eta)$.

Our first result concerns the entropy production for a PCA $(c f$. [4]). The corresponding result in continuous time has appeared in [9].

Proposition 2.1. Suppose $\mu$ is a stationary measure for a shift invariant, local PCA with transition probability $P(\mathrm{~d} \sigma \mid \eta)$. If $\mu$ is also a shift invariant Gibbs measure w.r.t. a certain potential $\Phi\left(\mu \in \mathcal{G}_{s}(\Phi)\right)$, then, for any shift invariant measure $\nu$,

$$
h(\nu \mid \mu)-h(P \nu \mid \mu)=\mathcal{H}_{\nu}\left(\hat{P}_{\nu} \mid \hat{P}_{\mu}\right)
$$

In particular, if $\nu \in \mathcal{G}_{s}(\Phi)$, then $P \nu \in \mathcal{G}_{s}(\Phi)$, that is the set of shift-invariant Gibbs measures w.r.t. the potential $\Phi$ is stable under the action of this PCA dynamics.

Proof. Let $\Lambda$ be a finite subset of $\mathbb{Z}^{d}$, and consider

$$
P_{\Lambda}(\sigma \mid \eta)=\prod_{i \in \Lambda} p_{i}\left(\sigma_{i} \mid \eta\right)
$$

This expression depends on the restriction of $\eta$ to a neighborhood of $\Lambda$, that we denote by $\bar{\Lambda}$.

Consider now the measure $Q_{\nu}(\mathrm{d} \sigma, \mathrm{d} \eta)$ defined above. For $A, B \subset \mathbb{Z}^{d}$ with $A$ finite, we denote by $Q_{\nu}\left(\sigma_{A} \mid \eta_{B}\right)$ the restriction to the $\sigma$-field generated by the projection $(\sigma, \eta) \rightarrow \sigma_{A}$ of the measure $Q$ conditioned to the $\sigma$-field generated by the projection $(\sigma, \eta) \rightarrow \eta_{B}$. So, e.g., $P_{\Lambda}(\sigma \mid \eta)=Q_{\nu}\left(\sigma_{\Lambda} \mid \eta_{\mathbb{Z}^{d}}\right) \equiv Q_{\nu}\left(\sigma_{\Lambda} \mid \eta\right)$, independently of $\nu$. Similarly, $\hat{Q}_{\nu}\left(\eta_{A} \mid \sigma_{B}\right)$ denotes the time-reversed conditioning, so that

$$
\pi_{\Lambda} \hat{P}_{\nu}\left(\eta_{\Lambda} \mid \sigma\right)=\hat{Q}_{\nu}\left(\eta_{\Lambda} \mid \sigma\right)
$$

For $C \subset \mathbb{Z}^{d}$ we will also use conditionings of the form

$$
\hat{Q}\left(\eta_{A} \mid \sigma_{B}, \eta_{C}\right)
$$

with the obvious meaning.

A simple computation, using the fact that $P \mu=\mu$, yields

$$
\begin{aligned}
h_{\bar{\Lambda}}(\nu \mid \mu)-h_{\Lambda}(P \nu \mid \mu) & =\sum_{\sigma_{\Lambda}} \pi_{\Lambda}(P \nu)\left(\sigma_{\Lambda}\right) \sum_{\eta_{\bar{\Lambda}}} \hat{Q}_{\nu}\left(\eta_{\bar{\Lambda}} \mid \sigma_{\Lambda}\right) \log \frac{\hat{Q}_{\nu}\left(\eta_{\bar{\Lambda}} \mid \sigma_{\Lambda}\right)}{\hat{Q}_{\mu}\left(\eta_{\bar{\Lambda}} \mid \sigma_{\Lambda}\right)} \\
& =E^{Q}\left[\log \frac{\hat{Q}_{\nu}\left(\eta_{\bar{\Lambda}} \mid \sigma_{\Lambda}\right)}{\hat{Q}_{\mu}\left(\eta_{\bar{\Lambda}} \mid \sigma_{\Lambda}\right)}\right] .
\end{aligned}
$$


Since

$$
h(\nu \mid \mu)-h(P \nu \mid \mu)=\lim _{\Lambda \uparrow \mathbb{Z}^{d}} \frac{1}{|\Lambda|}\left[h_{\Lambda}(\nu \mid \mu)-h_{\Lambda}(P \nu \mid \mu)\right]
$$

then the conclusion follows provided we show (see (2.4))

$$
\lim _{\Lambda \uparrow \mathbb{Z}^{d}} \frac{1}{|\Lambda|} E^{Q}\left[\log \frac{\hat{Q}_{\nu}\left(\eta_{\bar{\Lambda}} \mid \sigma_{\Lambda}\right)}{\hat{Q}_{\nu}\left(\eta_{\bar{\Lambda}} \mid \sigma\right)}\right]=0
$$

and

$$
\lim _{\Lambda \uparrow \mathbb{Z}^{d}} \frac{1}{|\Lambda|} E^{Q}\left[\log \frac{\hat{Q}_{\mu}\left(\eta_{\bar{\Lambda}} \mid \sigma_{\Lambda}\right)}{\hat{Q}_{\mu}\left(\eta_{\bar{\Lambda}} \mid \sigma\right)}\right]=0
$$

Note that (2.6) is a special case of (2.5).

Let now $\lambda^{\otimes}$ be the probability measure on $S^{\mathbb{Z}^{d}}$ obtained by taking the infinite product of the uniform measure $\lambda$ in $S$. We denote by $\lambda^{\otimes}\left(\sigma_{\Lambda}\right)$ the projection of $\lambda^{\otimes}$ on $\mathcal{F}_{\Lambda}$. Let also $\left\{i_{1}, \ldots, i_{|\bar{\Lambda}|}\right\}$ be the lexicographic ordering of the elements of $\bar{\Lambda}$; define $\bar{\Lambda}_{k}=\left\{i_{1}, \ldots, i_{k}\right\}$ for $1 \leq k \leq|\bar{\Lambda}|$, and $\bar{\Lambda}_{0}=\emptyset$. By the chain rule for conditional measures

$$
\log \frac{\hat{Q}\left(\eta_{\bar{\Lambda}} \mid \sigma_{\Lambda}\right)}{\lambda^{\otimes}\left(\eta_{\bar{\Lambda}}\right)}=\sum_{k=1}^{|\bar{\Lambda}|} \log \frac{\hat{Q}\left(\eta_{i_{k}} \mid \sigma_{\Lambda}, \eta_{\bar{\Lambda}_{k-1}}\right)}{\lambda\left(\eta_{i_{k}}\right)}
$$

Moreover, by shift invariance of $Q$

$$
E^{Q}\left[\log \frac{\hat{Q}\left(\eta_{i_{k}} \mid \sigma_{\Lambda}, \eta_{\bar{\Lambda}_{k-1}}\right)}{\lambda\left(\eta_{i_{k}}\right)}\right]=E^{Q}\left[\log \frac{\hat{Q}\left(\eta_{0} \mid \sigma_{\theta_{-i_{k}} \Lambda}, \eta_{\theta_{-i_{k}} \bar{\Lambda}_{k-1}}\right)}{\lambda\left(\eta_{0}\right)}\right]
$$

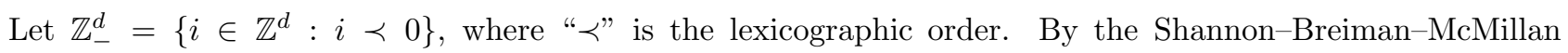
theorem [1], for every $\epsilon>0$ there are $A \subset \mathbb{Z}^{d}, B \subset \mathbb{Z}_{-}^{d}$ finite such that if $A \subset V$ and $B \subset W \subset \mathbb{Z}_{-}^{d}$ then

$$
\left|E^{Q}\left[\log \frac{\hat{Q}\left(\eta_{0} \mid \sigma_{V}, \eta_{W}\right)}{\lambda\left(\eta_{0}\right)}\right]-E^{Q}\left[\log \frac{\hat{Q}\left(\eta_{0} \mid \sigma_{A}, \eta_{B}\right)}{\lambda\left(\eta_{0}\right)}\right]\right|<\epsilon
$$

Note that, if we take $\Lambda$ large enough and $i_{k} \in \Lambda$ is far enough from the boundary of $\Lambda$, then $A \subset \theta_{-i_{k}} \Lambda$, and $B \subset \theta_{-i_{k}} \bar{\Lambda}_{k-1}$. For the other values of $i_{k} \in \bar{\Lambda}$,

$$
E^{Q}\left[\log \frac{\hat{Q}\left(\eta_{0} \mid \sigma_{\theta_{-i_{k}} \Lambda}, \eta_{\theta_{-i_{k}} \bar{\Lambda}_{k-1}}\right)}{\lambda\left(\eta_{0}\right)}\right] \leq \log |S|,
$$

which is the upper bound for the entropy of any probability measure is $S$ with respect to $\lambda$. Summing all up

$$
\lim _{\Lambda \uparrow \mathbb{Z}^{d}} \frac{1}{|\Lambda|} E^{Q}\left[\log \frac{\hat{Q}\left(\eta_{\bar{\Lambda}} \mid \sigma_{\Lambda}\right)}{\lambda^{\otimes}\left(\eta_{\bar{\Lambda}}\right)}\right]=E^{Q}\left[\log \frac{\hat{Q}\left(\eta_{0} \mid \sigma_{\mathbb{Z}^{d}}, \eta_{\mathbb{Z}_{-}^{d}}\right)}{\lambda\left(\eta_{0}\right)}\right] .
$$


Exactly in the same way one shows that

$$
\lim _{\Lambda \uparrow \mathbb{Z}^{d}} \frac{1}{|\Lambda|} E^{Q}\left[\log \frac{\hat{Q}\left(\eta_{\bar{\Lambda}} \mid \sigma\right)}{\lambda^{\otimes}\left(\eta_{\bar{\Lambda}}\right)}\right]=E^{Q}\left[\log \frac{\hat{Q}\left(\eta_{0} \mid \sigma_{\mathbb{Z}^{d}}, \eta_{\mathbb{Z}_{-}^{d}}\right)}{\lambda\left(\eta_{0}\right)}\right] .
$$

Thus (2.10) and (2.11) establish (2.5).

Next result shows that the measures in $\mathcal{P}_{s}$ for which the entropy production is zero are exactly those in $\mathcal{G}_{s}(\Phi)$. This result goes back to [10], where it has been proved for reversible systems in continuous time. The assumption of reversibility has been dropped in [12]. In discrete-time, the proof for a special class of reversible PCA is given in [11], Proposition 1. In the generality given here, the first proof was contained (but unpublished) in one of the authors' Ph.D. Thesis [4]. Later, a proof using general entropy arguments was given in [19]. In this paper we have preferred to emphasize the fact that the following result comes from the precise entropy production formula presented in Proposition 2.1.

Proposition 2.2. Under the same assumptions of Proposition 2.1, suppose $\nu \in \mathcal{P}_{s}$ is such that

$$
h(\nu \mid \mu)=h(P \nu \mid \mu)
$$

(in particular, this happens when $\nu$ is stationary). Then $\nu \in \mathcal{G}_{s}(\Phi)$.

Proof. By what seen in Proposition 2.1, equation (2.12) amounts to

$$
\mathcal{H}_{\nu}\left(\hat{P}_{\nu} \mid \hat{P}_{\mu}\right)=0
$$

We now adapt a classical argument for Gibbs measures (see e.g. [22], Th. 7.4). Let $V$ be a fixed hypercube and, for $k>0$,

$$
\partial_{k} V=\left\{i \in V^{c}: \operatorname{dist}(i, V) \leq k\right\},
$$

where dist $(\cdot)$ is the Euclidean distance. Take, now, a hypercube $\Lambda_{m, k}$ that is obtained as disjoint union of $m^{d}$ translates of $V \cup \partial_{k} V$, say

$$
\Lambda_{m, k}=\cup_{i=1}^{m^{d}} W_{i, k},
$$

where $W_{i, k}=T_{i}\left(V \cup \partial_{k} V\right)$, and $T_{i}$ is a suitable translation. We also write $V_{i}=T_{i} V$. Defining, for $i \in\left\{1, \ldots, m^{d}\right\}$

$$
B_{i, k}=W_{i, k} \backslash V_{i}
$$

we have (we use the notations introduced in the proof of Prop. 2.1)

$$
\log \frac{\hat{Q}_{\nu}\left(\eta_{\Lambda_{m, k}} \mid \sigma\right)}{\hat{Q}_{\mu}\left(\eta_{\Lambda_{m, k}} \mid \sigma\right)}=\sum_{i=1}^{m^{d}} \log \frac{\hat{Q}_{\nu}\left(\eta_{V_{i}} \mid \eta_{B_{i, k}}, \sigma\right)}{\hat{Q}_{\mu}\left(\eta_{V_{i}} \mid \eta_{B_{i, k}}, \sigma\right)}+\log \frac{\hat{Q}_{\nu}\left(\eta_{B_{1, k}} \mid \sigma\right)}{\hat{Q}_{\mu}\left(\eta_{B_{1, k}} \mid \sigma\right)}
$$

By positivity of relative entropy:

$$
E^{Q}\left[\log \frac{\hat{Q}_{\nu}\left(\eta_{B_{1, k}} \mid \sigma\right)}{\hat{Q}_{\mu}\left(\eta_{B_{1, k}} \mid \sigma\right)}\right] \geq 0
$$


so that

$$
E^{Q}\left[\log \frac{\hat{Q}_{\nu}\left(\eta_{\Lambda_{m, k}} \mid \sigma\right)}{\hat{Q}_{\mu}\left(\eta_{\Lambda_{m, k}} \mid \sigma\right)}\right] \geq \sum_{i=1}^{m^{d}} E^{Q}\left[\log \frac{\hat{Q}_{\nu}\left(\eta_{V_{i}} \mid \eta_{B_{i, k}}, \sigma\right)}{\hat{Q}_{\mu}\left(\eta_{V_{i}} \mid \eta_{B_{i, k}}, \sigma\right)}\right] .
$$

By translation invariance of $Q$ :

$$
E^{Q}\left[\log \frac{\hat{Q}_{\nu}\left(\eta_{V_{i}} \mid \eta_{B_{i, k}}, \sigma\right)}{\hat{Q}_{\mu}\left(\eta_{V_{i}} \mid \eta_{B_{i, k}}, \sigma\right)}\right]=E^{Q}\left[\log \frac{\hat{Q}_{\nu}\left(\eta_{V} \mid \eta_{T_{i}^{-1} B_{i, k}}, \sigma\right)}{\hat{Q}_{\mu}\left(\eta_{V} \mid \eta_{T_{i}^{-1} B_{i, k}}, \sigma\right)}\right] .
$$

Moreover, since $B_{i, k} \uparrow V_{i}^{c}$ as $k \uparrow+\infty$, using again the Shannon-Breiman-McMillan theorem, for each $\epsilon>0$ we can choose $k$ large enough so that

$$
\left|E^{Q}\left[\log \frac{\hat{Q}_{\nu}\left(\eta_{V} \mid \eta_{T_{i}^{-1} B_{i, k}}, \sigma\right)}{\hat{Q}_{\mu}\left(\eta_{V} \mid \eta_{T_{i}^{-1} B_{i, k}}, \sigma\right)}\right]-E^{Q}\left[\log \frac{\hat{Q}_{\nu}\left(\eta_{V} \mid \eta_{V^{c}}, \sigma\right)}{\hat{Q}_{\mu}\left(\eta_{V} \mid \eta_{V^{c}}, \sigma\right)}\right]\right| \leq \epsilon .
$$

Summing up $(2.14,2.15)$ and $(2.16)$, we get

$$
\frac{1}{m^{d}} E^{Q}\left[\log \frac{\hat{Q}_{\nu}\left(\eta_{\Lambda_{m, k}} \mid \sigma\right)}{\hat{Q}_{\mu}\left(\eta_{\Lambda_{m, k}} \mid \sigma\right)}\right] \geq E^{Q}\left[\log \frac{\hat{Q}_{\nu}\left(\eta_{V} \mid \eta_{V^{c}}, \sigma\right)}{\hat{Q}_{\mu}\left(\eta_{V} \mid \eta_{V^{c}}, \sigma\right)}\right]-\epsilon .
$$

But $m^{d}$ is proportional to $\left|\Lambda_{m, k}\right|$, so, by $(2.13)$

$$
\lim _{m \rightarrow+\infty} \frac{1}{m^{d}} E^{Q}\left[\log \frac{\hat{Q}_{\nu}\left(\eta_{\Lambda_{m, k}} \mid \sigma\right)}{\hat{Q}_{\mu}\left(\eta_{\Lambda_{m, k}} \mid \sigma\right)}\right]=0 .
$$

Thus, since $\epsilon$ is arbitrary, equation (2.17) yields

$$
E^{Q}\left[\log \frac{\hat{Q}_{\nu}\left(\eta_{V} \mid \eta_{V^{c}}, \sigma\right)}{\hat{Q}_{\mu}\left(\eta_{V} \mid \eta_{V^{c}}, \sigma\right)}\right]=0
$$

that, by elementary properties of relative entropy, implies

$$
\hat{Q}_{\nu}\left(\eta_{V} \mid \eta_{V^{c}}, \sigma\right)=\hat{Q}_{\mu}\left(\eta_{V} \mid \eta_{V^{c}}, \sigma\right) \quad Q-\text { a.s. }
$$

At this point we use Proposition 3.2 in [13], which implies that if (2.18) holds for a $\mu \in \mathcal{G}_{s}(\Phi)$, then $\nu\left(\eta_{V} \mid \eta_{V^{c}}\right)$ $=\mu\left(\eta_{V} \mid \eta_{V^{c}}\right)$ a.s. and then $\nu \in \mathcal{G}_{s}(\Phi)$ too. This completes the proof.

\section{A CLASS OF REVERSible DYNAMiCS}

In this section we introduce a class of reversible PCA's we will be dealing with in the rest of the paper, and give some general results on their stationary measures, resp. reversible measures. Let us remember that a PCA $P$ is called reversible if there exists at least one probability measure $\mu$ such that the Markov process with initial law $\mu$ and dynamics $P$ is reversible.

We choose $S=\{-1,1\}$ as spin space. Consider a function $k: \mathbb{Z}^{d} \rightarrow \mathbb{R}$ that is of finite range, i.e. there exists $R>0$ such that $k(i)=0$ for $|i|>R$, and symmetric, i.e. $k(i)=k(-i)$ for every $i \in \mathbb{Z}^{d}$ (this last assumption being necessary to assure the reversibility of the PCA, $c f$. [11]). Moreover, let $\tau \in\{-1,1\}^{\mathbb{Z}^{d}}$ be 
a fixed configuration, that will play the role of boundary condition. For $\Lambda \subset \mathbb{Z}^{d}$, we define the transition probability $P_{\Lambda}^{\tau}(\mathrm{d} \sigma \mid \eta)=\otimes_{i \in \Lambda} P_{i}^{\tau}\left(\mathrm{d} \sigma_{i} \mid \eta\right)$ by

$$
P_{i}^{\tau}\left(\sigma_{i}=s \mid \eta\right)=p_{i}(s \mid \tilde{\eta})=\frac{1}{2}\left[1+s \tanh \left(\beta \sum_{j \in \mathbb{Z}^{d}} k(i-j) \tilde{\eta}_{j}+\beta h\right)\right],
$$

where $\tilde{\eta}=\eta_{\Lambda} \tau_{\Lambda^{c}} ; h \in \mathbb{R}, \beta>0$ are given parameters. According to [11], this particular form of $p_{i}$ is indeed the most general one for a shift invariant non degenerate local reversible PCA on $\{-1,1\}^{\mathbb{Z}^{d}}$.

In the case $\Lambda$ is a hypercube, we can also consider periodic boundary conditions. The associated transition probability is denoted by $P_{\Lambda}^{\text {per }}$. In general, when $\Lambda$ is finite, we write $P_{\Lambda}^{\tau}(\sigma \mid \eta)$ in place of $P_{\Lambda}^{\tau}(\{\sigma\} \mid \eta)$. In the case $\Lambda=\mathbb{Z}^{d}$, the boundary condition $\tau$ plays no role, and will be omitted.

In the rest of this section we establish some facts about stationary and reversible measures for these PCA's.

Proposition 3.1. Let $\Lambda \subset \mathbb{Z}^{d}$ finite, and $\tau \in\{-1,1\}^{\mathbb{Z}^{d}}$. Then the finite volume PCA with transition probability $P_{\Lambda}^{\tau}(\sigma \mid \eta)$ has a unique stationary measure $\nu_{\Lambda}^{\tau}$ given by

$$
\nu_{\Lambda}^{\tau}(\sigma)=\frac{1}{W_{\Lambda}^{\tau}} \prod_{i \in \Lambda} \mathrm{e}^{\beta h \sigma_{i}} \cosh \left[\beta \sum_{j \in \mathbb{Z}^{d}} k(i-j) \tilde{\sigma}_{j}+\beta h\right] \mathrm{e}^{\beta \sigma_{i} \sum_{j \in \Lambda^{c}} k(i-j) \tau_{j}},
$$

where, as before, $\tilde{\sigma}=\sigma_{\Lambda} \tau_{\Lambda^{c}}$, and $W_{\Lambda}^{\tau}$ is the normalization. Moreover, $\nu_{\Lambda}^{\tau}$ is reversible for $P_{\Lambda}^{\tau}$.

Proof. It is clear that $P_{\Lambda}^{\tau}(\sigma \mid \eta)>0 \forall \sigma, \eta$, so that the Markov chain with transition probability $P_{\Lambda}^{\tau}$ has a unique stationary measure. Thus, we only have to show that $\nu_{\Lambda}^{\tau}$ is reversible, i.e.

$$
P_{\Lambda}^{\tau}(\sigma \mid \eta) \nu_{\Lambda}^{\tau}(\eta) \equiv P_{\Lambda}^{\tau}(\eta \mid \sigma) \nu_{\Lambda}^{\tau}(\sigma)
$$

Observe that, since $\sigma_{i} \in\{-1,1\}, P_{\Lambda}^{\tau}$ may be written in the form

$$
P_{\Lambda}^{\tau}(\sigma \mid \eta)=\prod_{i \in \Lambda} \frac{\mathrm{e}^{\beta \sigma_{i}\left(\sum_{j} k(i-j) \tilde{\eta}_{j}+h\right)}}{2 \cosh \left(\beta \sum_{j} k(i-j) \tilde{\eta}_{j}+\beta h\right)} .
$$

Thus (3.2) amounts to

$$
\sum_{i \in \Lambda} \sum_{j \in \mathbb{Z}^{d}} \sigma_{i} \tilde{\eta}_{j} k(i-j)+\sum_{i \in \Lambda} \sum_{j \notin \Lambda} \eta_{i} \tau_{j} k(i-j)=\sum_{i \in \Lambda} \sum_{j \in \mathbb{Z}^{d}} \eta_{i} \tilde{\sigma}_{j} k(i-j)+\sum_{i \in \Lambda} \sum_{j \notin \Lambda} \sigma_{i} \tau_{j} k(i-j)
$$

which is easily checked.

The above result on stationary measures for PCA's in finite volume, has an immediate consequence in infinite volume.

Proposition 3.2. Let $\tau$ be any fixed boundary condition, and $\mu$ be any limit point of the $P_{\Lambda}^{\tau}$-stationary measure $\nu_{\Lambda}^{\tau}$ as $\Lambda \uparrow \mathbb{Z}^{d}$. Then $\mu$ is reversible for the infinite volume $P C A P(\mathrm{~d} \sigma \mid \eta)=\otimes_{i} P_{i}\left(\mathrm{~d} \sigma_{i} \mid \eta\right)$ where $P_{i}$ is defined by

$$
P_{i}\left(\sigma_{i}=s \mid \eta\right)=\frac{1}{2}\left[1+s \tanh \left(\beta \sum_{j \in \mathbb{Z}^{d}} k(i-j) \eta_{j}+\beta h\right)\right],
$$


and $\mu$ is Gibbsian for the shift-invariant potential $\Phi$ given by

$$
\begin{aligned}
& \Phi_{\{i\}}\left(\sigma_{i}\right)=-\beta h \sigma_{i} \\
& \Phi_{U_{i}}\left(\sigma_{U_{i}}\right)=-\log \cosh \left[\beta \sum_{j} k(i-j) \sigma_{j}+\beta h\right] \\
& \Phi_{\Lambda}\left(\sigma_{\Lambda}\right)=0 \text { otherwise, }
\end{aligned}
$$

where $U_{i}=\{j: k(i-j) \neq 0\}$, that is finite by assumption.

Proof. Note that the finite volume Gibbs measure for $\Phi$ is

$$
\mu_{\Lambda}^{\tau}(\sigma)=\frac{1}{Z_{\Lambda}^{\tau}} \prod_{i: \operatorname{dist}(i, \Lambda) \leq R} \cosh \left[\beta \sum_{j} k(i-j) \tilde{\sigma}_{j}+\beta h\right] \mathrm{e}^{\beta h \sigma_{i}}
$$

that differs from $\nu_{\Lambda}^{\tau}$ only for boundary terms (and for the renormalization constant). The fact that the limit of $\nu_{\Lambda}^{\tau}$ is Gibbsian for $\Phi$ follows therefore from general facts on Gibbs measures [6]. The reversibility of $\mu$ for the infinite volume PCA is obtained as follows. Let $f:\{-1,1\}^{\mathbb{Z}^{d}} \times\{-1,1\}^{\mathbb{Z}^{d}} \rightarrow \mathbb{R}$ be a function which is local in both variables. For $\Lambda$ large enough, reversibility of $\nu_{\Lambda}^{\tau}$ yields

$$
\sum_{\sigma, \tau} P_{\Lambda}^{\tau}(\sigma \mid \eta) \nu_{\Lambda}^{\tau}(\eta) f(\sigma, \eta)=\sum_{\sigma, \tau} P_{\Lambda}^{\tau}(\eta \mid \sigma) \nu_{\Lambda}^{\tau}(\sigma) f(\sigma, \eta)
$$

Note that, for $\Lambda$ large enough, the boundary condition $\tau$ in $P_{\Lambda}^{\tau}$ does not play any role in (3.5). Thus, letting $\Lambda \uparrow \mathbb{Z}^{d}$ in (3.5) obtaining

$$
\int P(\mathrm{~d} \sigma \mid \eta) \mu(\mathrm{d} \eta) f(\sigma, \eta)=\int P(\mathrm{~d} \eta \mid \sigma) \mu(\mathrm{d} \sigma) f(\sigma, \eta)
$$

that establishes reversibility of $\mu$.

\section{Remark 3.1.}

- Instead of fixed boundary conditions, one can choose periodic boundary conditions. In this case, the finite volume measure defined by

$$
\nu_{\Lambda}^{\mathrm{per}}(\sigma)=\frac{1}{W_{\Lambda}^{\mathrm{per}}} \prod_{i \in \Lambda} \cosh \left[\beta \sum_{j \in \mathbb{Z}^{d}} k(i-j) \tilde{\sigma}_{j}+\beta h\right] \mathrm{e}^{\beta h \sigma_{i}}
$$

where $\tilde{\sigma}$ is the periodic continuation of $\sigma$, is the unique stationary reversible measure for $P_{\Lambda}^{\text {per }}$. Remark that, in opposition to fixed boundary conditions, we now have that $\nu_{\Lambda}^{\text {per }}=\mu_{\Lambda}^{\text {per }}$, which means that the finite volume stationary measure for the finite volume PCA is equal to the local specification of the associated Gibbs measure.

- As follows from the results in [11], the potentials described in (3.4) are the only ones on $\{-1,1\}^{\mathbb{Z}^{d}}$ for which the associated Gibbs measures may be reversible for a local, shift invariant, non degenerate PCA. Note that, even if for all $|j|>1, k(j)=0$ (i.e. the range of $k$ is 1 ), the range of the interaction $\Phi$ is at least 2. This illustrates Theorem 4.2 of [5], which ensures that Gibbs measures associated to potentials with nearest neighbor interaction cannot be reversible for a PCA.

Moreover, the following result gives a complete description of the links between the set of reversible measures for the PCA $P$ (which will be denoted by $\mathcal{R}$ ), the set of stationary ones denoted by $\mathcal{S}$, the set $\mathcal{G}(\Phi)$ of Gibbs measures with respect to the potential $\Phi$ defined by (3.4), and their respective intersections with the set of shift-invariant measures: $\mathcal{R}_{s}, \mathcal{S}_{s}, \mathcal{G}_{s}(\Phi)$. 
Proposition 3.3. The reversible measures for the PCA P defined in (3.1) are exactly those Gibbs measures w.r.t. $\Phi$ given in (3.4) which are also stationary:

$$
\mathcal{R}=\mathcal{S} \cap \mathcal{G}(\Phi)
$$

Moreover, the subset of shift invariant reversible measures is equal to the set of shift invariant stationary measures:

$$
\mathcal{R}_{s}=\mathcal{S}_{s}
$$

Proof. The proof of the first assertion is based on the following proposition proved in [11]:

Let $P$ be a non degenerate local reversible PCA. Each reversible measure $\mu$ for $P$ is Gibbs w.r.t. a certain potential $\Phi_{P}$. Reciprocally, any Gibbs measure w.r.t. $\Phi_{P}$ is either a reversible measure for $P$ or periodic of period two.

Since obviously $\mathcal{R} \subset \mathcal{S}$, the abovementioned proposition implies $\mathcal{R} \subset \mathcal{S} \cap \mathcal{G}(\Phi)$. For the reciprocal inclusion, since stationary measures can not be 2-periodic, a stationary Gibbsian measure is necessarily a reversible one.

To prove the second assertion, note that by Proposition 3.2 and Remark 3.1, $\mathcal{S}_{s} \cap \mathcal{G}_{s}(\Phi) \ni \mu^{\text {per }}$. Thus Proposition 2.2 applies, that is: $\mathcal{S}_{s} \subset \mathcal{G}_{s}(\Phi)$. On the other hand, from the first assertion: $\mathcal{R}_{s}=\mathcal{S}_{s} \cap \mathcal{G}_{s}(\Phi)$. Then $\mathcal{R}_{s}=\mathcal{S}_{s}$.

Remark 3.2. The proof of Proposition 3.3 does not use the specific form of the PCA P. So equalities (3.7) and (3.8) hold as soon as Proposition 2.2 and the abovementioned result of [11] apply, that is for the general class of local, shift invariant, non degenerate reversible PCA dynamics on $S^{\mathbb{Z}^{d}}$ for any $S$ finite.

\section{Phase transition}

In this section we show that for some reversible PCA it is indeed the case that not all Gibbs measures for the potential in (3.4) are stationary. We treat those PCA defined in (3.1) for which $k(i)=0$ for $|i|>1$ (i.e. $R=1), h=0$ and $d=2$. Besides $\beta$, there are three parameters in the game: $k(0), k\left(e_{1}\right)$ and $k\left(e_{2}\right)$, where $e_{1}, e_{2}$ are the basis vectors in $\mathbb{R}^{2}$. The first result concerns the existence of phase transition for the potential $\Phi$.

Proposition 4.1. Assume $k\left(e_{1}\right) \neq 0, k\left(e_{2}\right) \neq 0$. Then there exists $\beta_{c} \in(0,+\infty)$ such that for $\beta>\beta_{c}$ $|\mathcal{G}(\Phi)|>1$.

Proof. We divide the proof into different cases, depending on the signs of $k(0), k\left(e_{1}\right), k\left(e_{2}\right)$. Note that the transformation $k(\cdot) \rightarrow-k(\cdot)$ leaves invariant the potential $\Phi$.

Case 1: $k(0) \geq 0, k\left(e_{1}\right)>0, k\left(e_{2}\right)>0$.

For a given square $\Lambda \subset \mathbb{Z}^{2}$, let $C l_{m}(\Lambda)=\left\{i \in \mathbb{Z}^{d}\right.$ : $\left.\operatorname{dist}(i, \Lambda) \leq m\right\}$. Consider a fixed configuration $\sigma \in\{-1,+1\}^{\mathbb{Z}^{2}}$ such that $\sigma_{i} \equiv+1$ for $i \notin \Lambda\left(\sigma_{\Lambda^{c}} \equiv+1\right)$. Moreover let $\mathbb{Z}_{*}^{2}=\mathbb{Z}^{2}+(1 / 2,1 / 2)$. We recall the classical notion of Peierls contour associated to $\sigma$. We say that the segment joining two nearest neighbors $a, b \in \mathbb{Z}_{*}^{2}$ is marked if this segment separates two nearest neighbors $i, j \in \mathbb{Z}^{2}$ for which $\sigma_{i} \sigma_{j}=-1$. Marked segments form a finite family of closed, non self-intersecting, piecewise linear curves, that we call Peierls contours. Each segment of a contour $\gamma$ separates two nearest neighbors whose spins have different signs (they necessarily belong to $C l_{1}(\Lambda)$ ). If $i, j$ are nearest neighbors separated by $\gamma$ and $\sigma_{i}=-1$ we write $i \in \partial^{-} \gamma$ and $j \in \partial^{+} \gamma$. We call the union of the sets of sites $\partial^{-} \gamma$ and $\partial^{+} \gamma$ the boundary of the contour $\gamma$. For each $i \in \Lambda$ for which $\sigma_{i}=-1$, there is a minimal Peierls contour $\gamma$ around $i$, i.e. such that $i$ is in the interior of the closed curve $\gamma$.

This notion of minimal contour is the one used for the Ising model. Here we have to modify it as follows. Two Peierls contours $\gamma, \gamma^{\prime}$ are called adjacent if their boundaries have a common point. We say that two Peierls contours $\gamma, \gamma^{\prime}$ communicates if they belong to a sequence of Peierls contours $\gamma_{1}, \ldots, \gamma_{n}$ such that for all $k, \gamma_{k}$ and $\gamma_{k+1}$ are adjacent. The relation of communicating is an equivalence relation. We call simply contour the union of the Peierls contours in an equivalence class. The minimal contour around $i$ with $\sigma_{i}=-1$ is the one 


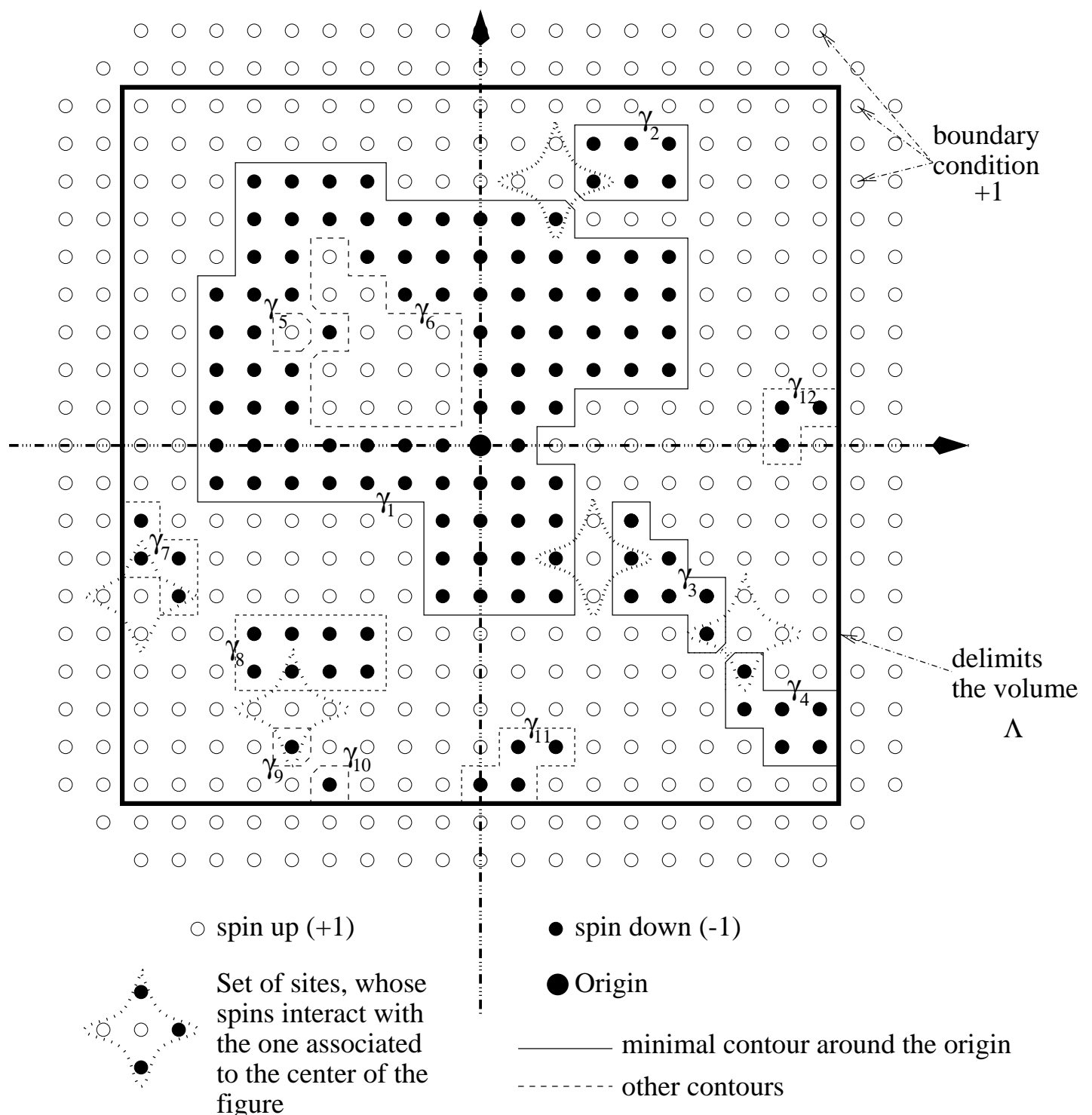

Figure 1. Example of a configuration $\sigma$ on $C l_{2}(\Lambda)$ such that $\sigma_{0}=-1$ and $\sigma_{\Lambda^{c}} \equiv+1$. Drawing of its corresponding contours: $\gamma_{1}$ is the minimal Peierls contour around the origin; $\left(\gamma_{1} \cup \gamma_{2} \cup \gamma_{3} \cup \gamma_{4}\right)$ is the minimal contour around the origin (i.e. the equivalence class of $\left.\gamma_{1}\right)$; $\left\{\gamma_{5}, \gamma_{6}\right\},\left\{\gamma_{7}\right\},\left\{\gamma_{8}, \gamma_{9}, \gamma_{10}\right\},\left\{\gamma_{11}\right\},\left\{\gamma_{12}\right\}$ are the other equivalence classes.

formed by the equivalence class which contains the minimal Peierls contour around $i$. The boundary $\left(\partial^{+}\right.$or $\partial^{-}$) of a contour is simply the union of the boundaries of the Peierls contours that form it (see Fig. 1).

Let now $\mu_{\Lambda}^{+}$be the finite volume Gibbs measure with + boundary condition, that we write as follows:

$$
\mu_{\Lambda}^{+}(\sigma)=\frac{1}{\mathbb{Z}_{\Lambda}^{+}} \prod_{i \in C l_{1}(\Lambda)} \frac{\cosh \left(\beta \sum_{j} k(i-j) \sigma_{j}^{+}\right)}{\cosh \left(\beta \sum_{j} k(i-j)\right)} \quad \text { with } \sigma^{+}=\sigma_{\Lambda}(+1)_{\Lambda^{c}} .
$$


We have modified the normalization for later convenience. A given $\sigma^{+} \in\{-1,+1\}^{\mathbb{Z}^{2}}$ corresponds, as described above, to a collection of contours $\Gamma=\left\{c_{1}, \ldots, c_{m}\right\}$. Each contour $c_{i}$ is a union of Peierls contours. Peierls contours belonging to different $c_{i}$ 's do not communicate. We can write:

$$
\mu_{\Lambda}^{+}(\sigma)=\frac{1}{\mathbb{Z}_{\Lambda}^{+}} \prod_{k=1}^{m} F\left(c_{k}\right),
$$

where

$$
F\left(c_{k}\right)=\prod_{i \in \partial c_{k}} \frac{\cosh \left(\beta \sum_{j} k(i-j) \sigma_{j}\right)}{\cosh \left(\beta \sum_{j} k(i-j)\right)}
$$

and $\partial c_{k}=\partial^{+} c_{k} \cup \partial^{-} c_{k}$. Observing that if $\sigma_{0}=-1$ then there is a contour around 0 , we have:

$$
\mu_{\Lambda}^{+}\left(\sigma_{0}=-1\right)=\frac{1}{\mathbb{Z}_{\Lambda}^{+}} \sum_{c_{1} \text { around } 0} F\left(c_{1}\right) \sum_{\Gamma \ni c_{1}} F\left(\Gamma \backslash c_{1}\right),
$$

where, for $\Gamma=c_{1} \cup c_{2} \cup \cdots \cup c_{m}$, we let $F\left(\Gamma \backslash c_{1}\right)=\prod_{k=2}^{m} F\left(c_{k}\right)$. Note that, if $\Gamma$ is a contour, $\Gamma \backslash c_{1}$ is also a contour, that corresponds to the configuration obtained by flipping all the spins -1 inside $c_{1}$ in the configuration associated to $\Gamma$. It follows that

$$
\sum_{\Gamma \ni c_{1}} F\left(\Gamma \backslash c_{1}\right) \leq Z_{\Lambda}^{+} \equiv \sum_{\Gamma} F(\Gamma)
$$

and therefore

$$
\mu_{\Lambda}^{+}\left(\sigma_{0}=-1\right) \leq \sum_{c_{1} \text { around } 0} F\left(c_{1}\right) .
$$

Now note that if $c_{1}$ is a contour and $i \in \partial c_{1}$, then the spins $\sigma_{i}, \sigma_{i \pm e_{1}}, \sigma_{i \pm e_{2}}$ do not have the same sign, so that

$$
\frac{\cosh \left(\beta \sum_{j} k(i-j) \sigma_{j}\right)}{\cosh \left(\beta \sum_{j} k(i-j)\right)} \leq \frac{\cosh (\beta A)}{\cosh (\beta B)},
$$

where $B=\sum_{j} k(j), A$ is the maximum value of $\left.\mid \sum_{j} k(i-j) \sigma_{j}\right) \mid$ for $\sigma$ such that $\sigma_{0}, \sigma_{ \pm e_{1}}, \sigma_{ \pm e_{2}}$ do not have the same sign, and therefore $A<B$. Thus, we have to compare for a contour $c_{1}$, the cardinal of its boundary $\left|\partial c_{1}\right|$ with its length denoted by $l\left(c_{1}\right)$. But remark that to any point of $\partial c_{1}$ correspond at most 4 marked segments on $c_{1}$. So, $l\left(c_{1}\right) \leq 4\left|\partial c_{1}\right|$, and we have

$$
F\left(c_{1}\right) \leq\left[\frac{\cosh (\beta A)}{\cosh (\beta B)}\right]^{\left|\partial c_{1}\right|} \leq\left[\frac{\cosh (\beta A)}{\cosh (\beta B)}\right]^{l\left(c_{1}\right) / 4} .
$$

On the other hand, for a given length $l$, it is easily checked that the number of contours around 0 of length $l$ is bounded by $l^{3} 3^{l-1}$. Thus, by (4.1),

$$
\mu_{\Lambda}^{+}\left(\sigma_{0}=-1\right) \leq \sum_{l \geq 0} l^{3} 3^{l-1}\left[\frac{\cosh (\beta A)}{\cosh (\beta B)}\right]^{l / 4}
$$

that goes to zero as $\beta \uparrow+\infty$. Thus, taking $\beta$ large enough and letting $\Lambda \uparrow \mathbb{Z}^{d}$ in $\mu_{\Lambda}^{+}$, we construct a Gibbs measure $\mu$ for which $\mu^{+}\left(\sigma_{0}=-1\right)<1 / 2$. Symmetrically, taking minus boundary conditions, we obtain a Gibbs measure $\mu^{-}$for which $\mu^{-}\left(\sigma_{0}=-1\right)>1 / 2$, and this proves phase transition. 
Case 2: $k(0)<0, k\left(e_{1}\right)>0, k\left(e_{2}\right)>0$.

Define

$$
k^{*}(i)= \begin{cases}k(i) & \text { for } i \neq 0 \\ -k(0) & \text { for } i=0\end{cases}
$$

and let $\Phi^{*}$ be the associated potential. Consider also the map $T:\{-1,1\}^{\mathbb{Z}^{2}} \rightarrow\{-1,1\}^{\mathbb{Z}^{2}}$ given by

$$
(T \sigma)_{i}= \begin{cases}\sigma_{i} & \text { for } i \in \mathbb{Z}_{e}^{2} \\ -\sigma_{i} & \text { for } i \in \mathbb{Z}_{o}^{2}\end{cases}
$$

To stress dependence on the potential $\Phi$ we write $\mu_{\Lambda, \Phi}^{\tau}$ for $\mu_{\Lambda}^{\tau}$. It is easily seen that

$$
\mu_{\Lambda, \Phi}^{\tau}(\sigma)=\mu_{\Lambda, \Phi^{*}}^{T \tau}(T \sigma)
$$

so that the map $\mu \rightarrow \mu \circ T^{-1}$ is a bijection between $\mathcal{G}(\Phi)$ and $\mathcal{G}\left(\Phi^{*}\right)$. The conclusion follows from the fact that $\left|\mathcal{G}\left(\Phi^{*}\right)\right|>1$, as seen in case 1 .

Case 3: $k(0) \geq 0, k\left(e_{1}\right)>0, k\left(e_{2}\right)<0$.

This case is treated as case 2 , with the following choices:

$$
k^{*}(i)= \begin{cases}k(i) & \text { for } i \neq e_{2} \\ -k\left(e_{2}\right) & \text { for } i=e_{2}\end{cases}
$$

and

$$
(T \sigma)_{i}= \begin{cases}\sigma_{i} & \text { for } i=(x, y) \text { with } y \text { even } \\ -\sigma_{i} & \text { otherwise. }\end{cases}
$$

the proof is now completed.

Remark 4.1. The special case $k(0)=0$ was already treated in [11] example 2 (for $\left.k\left(e_{1}\right)=k\left(e_{2}\right)=1\right)$, where a remarkable relation with Ising model was pointed out. We recall here in some more generality the principal steps of the argumentation: let $\mathbb{Z}_{o}^{2}=\left\{(x, y) \in \mathbb{Z}^{2}: x+y\right.$ is odd $\}, \mathbb{Z}_{e}^{2}=\mathbb{Z}^{2} \backslash \mathbb{Z}_{o}^{2}$ and, similarly, $\Lambda_{o}=\Lambda \cap \mathbb{Z}_{o}^{2}$, $\Lambda_{e}=\Lambda \cap \mathbb{Z}_{e}^{2}$. Note that since $k(0)=0, \sigma_{\Lambda_{o}}$ and $\sigma_{\Lambda_{e}}$ are independent under $\mu_{\Lambda}^{\tau}$, i.e. $\mu_{\Lambda}^{\tau}=\mu_{\Lambda_{e}}^{\tau} \otimes \mu_{\Lambda_{o}}^{\tau}$. Consider the following anisotropic Ising model on $\{-1,1\}^{\Lambda}$ :

$$
\rho_{\Lambda}^{\tau}(\sigma)=\frac{1}{N_{\Lambda}^{\tau}} \exp \left[\beta \sum_{i \in \Lambda}\left(k\left(e_{1}\right) \sigma_{i} \tilde{\sigma}_{i+e_{1}}+k\left(e_{2}\right) \sigma_{i} \tilde{\sigma}_{i+e_{2}}\right)\right]
$$

where $N_{\Lambda}^{\tau}$ is the normalization and $\tilde{\sigma}=\sigma_{\Lambda} \tau_{\Lambda^{c}}$. Restricting this measure to the sites in $\Lambda_{e}$ we obtain

$$
\begin{aligned}
\pi_{\Lambda_{e}} \rho_{\Lambda}^{\tau}\left(\sigma_{\Lambda_{e}}\right) & =\sum_{\sigma_{\Lambda_{o}}} \rho_{\Lambda}^{\tau}(\sigma) \\
& =\frac{2}{N_{\Lambda}^{\tau}} \prod_{i \in \Lambda_{o}} \cosh \left[\beta \sum_{j} k(i-j) \tilde{\sigma}_{j}\right] \\
& =\mu_{\Lambda_{o}}^{\tau}\left(\sigma_{\Lambda_{e}}\right) .
\end{aligned}
$$


Therefore, phase transition for $\mathcal{G}(\Phi)$ follows from phase transition for the Ising model: since $\rho^{-}\left(\sigma_{0}=+1\right)<$ $\frac{1}{2}<\rho^{+}\left(\sigma_{0}=+1\right)$, the restrictions $\pi_{\Lambda_{e}} \rho^{-}$and $\pi_{\Lambda_{e}} \rho^{+}$are different, and then

$$
\left(\mu_{\mathbb{Z}_{o}^{2}}^{+}=\pi_{\Lambda_{e}} \rho^{+}\right) \neq\left(\pi_{\Lambda_{e}} \rho^{-}=\mu_{\mathbb{Z}_{o}^{2}}^{-}\right)
$$

We now give an example of situation where, due to the phase transition, some elements of $\mathcal{G}(\Phi)$ are not stationary.

Proposition 4.2. Suppose $k(0) \leq 0, k\left(e_{1}\right)<0, k\left(e_{2}\right)<0$, and let $\mu^{+}$be the Gibbs measure corresponding to plus boundary conditions. Suppose $\beta$ is large enough so that $\mu^{+} \neq \mu^{-}$. Then $\mu^{+}$and $\mu^{-}$are not stationary.

Proof. We first observe that the transformation $k(\cdot) \rightarrow-k(\cdot)$ do not change the elements of $\mathcal{G}(\Phi)$, but it does change the dynamics. We recall few basic notions on stochastic ordering. Given $\sigma, \eta \in\{-1,1\}^{\mathbb{Z}^{2}}$, we say that $\sigma \leq \eta$ if $\sigma_{i} \leq \eta_{i}$ for every $i \in \mathbb{Z}^{2}$. Monotonicity of functions $\{-1,1\}^{\mathbb{Z}^{2}} \rightarrow \mathbb{R}$ is defined with respect to this partial order. Finally, for $\nu, \mu$ probabilities on $\{-1,1\}^{\mathbb{Z}^{2}}$, we say that $\nu \leq \mu$ if $\int f \mathrm{~d} \nu \leq \int f \mathrm{~d} \mu$ for every increasing $f$.

The key observation consists in the fact that, under our assumptions on $k(\cdot)$, the transition probability $P(\mathrm{~d} \sigma \mid \eta)$ is decreasing, i.e.

$$
\nu \leq \mu \text { implies } P \nu \geq P \mu \text {. }
$$

This follows from the facts that $p_{0}(1 \mid \eta)$ is decreasing in $\eta$, while $p_{0}(-1 \mid \eta)$ is increasing in $\eta$ (see [14] or [16] for details). Let now $\mu^{0}$ be a limit point of the sequence $\nu_{\Lambda}^{\text {per }}$ defined in Remark 3.1. By using the criterion in [15] (Th. II 2.9), it is easy to check that $\nu_{\Lambda}^{\text {per }} \leq \nu_{\Lambda}^{+}$for every $\Lambda$, and so $\mu^{0} \leq \mu^{+}$. Moreover, $\mu^{0}\left(\sigma_{0}=\right.$ $+1)=\frac{1}{2}<\mu^{+}\left(\sigma_{0}=+1\right)$. So $\mu^{0} \neq \mu^{+}$. On the other hand, by Proposition 3.2, $\mu^{0}$ is stationary. Therefore $P \mu^{+} \leq P \mu^{0}=\mu^{0}<\mu^{+}$, which completes the proof that $\mu^{+}$is not stationary. By symmetrical arguments, the same holds for $\mu^{-}$.

Remark 4.2. In fact, the above proposition is a particular case of more general situations. Let us present two of them:

- take $P$ a reversible PCA on $\{-1,+1\}^{\mathbb{Z}^{2}}$ with the same characteristics as in Proposition 4.2, except that the range of the symmetric negative interaction function $k$ is no more equal to 1 , but is finite. The same arguments as above apply to deduce that $P$ is decreasing and $\mu^{0} \leq \mu^{+}$, where $\mu^{0}$ and $\mu^{+}$are respectively the Gibbs measures corresponding to periodical boundary conditions and to +1 -boundary conditions. Furthermore $\mu^{0}$ is $P$-stationary. So, $P \mu^{+} \leq P \mu^{0}=\mu^{0} \leq \mu^{+}$. To conclude, it is enough to prove that $\mu^{0} \neq \mu^{+}$. This is clear remarking that, on one side, by Proposition $4.1, \mu^{0}\left(\sigma_{0}=+1\right)=\frac{1}{2}<\mu_{R=1}^{+}\left(\sigma_{0}=+1\right)$ where $\mu_{R=1}^{+}$is the + -Gibbs measure associated with a truncated version of the interaction $k$ defined by $k_{R=1}(i)=k(i) \mathbf{1}_{|i| \leq 1}$; on the other side, by monotonicity, $\mu_{R=1}^{+} \leq \mu^{+}$which implies $\mu_{R=1}^{+}\left(\sigma_{0}=+1\right) \leq$ $\mu^{+}\left(\sigma_{0}=+1\right)$. So, $\mu^{+}$is not stationary for $P$;

- let us now present a more general situation involving PCA's that are possibly non-reversible. Take $P$ a $\mathrm{PCA}$ on $\{-1,+1\}^{\mathbb{Z}^{d}}$ whose transition probability is increasing, i.e. it preserves the order of probability measures. This implies in particular (cf. [23], Th. 18.13) that, when the initial condition is the deterministic configuration equal to the constant +1 (respectively -1 ), the PCA converges, as time goes to infinity, towards a $P$-stationary measure $\nu^{+}$(resp. $\left.\nu^{-}\right)$.

Let now $T$ be the map on $\{-1,1\}^{\mathbb{Z}^{d}}$ which changes the sign of each spin: $(T \sigma)_{i}=-\sigma_{i}, i \in \mathbb{Z}^{d}$, and assume the following identity holds:

$$
P(\mathrm{~d} \sigma \mid \eta) \circ T^{-1}=P(\mathrm{~d} \sigma \mid T \eta) .
$$


An example of such attractive PCA is given by $P(\mathrm{~d} \sigma \mid \eta)=\otimes_{i} P_{i}\left(\mathrm{~d} \sigma_{i} \mid \eta\right)$ where $P_{i}$ is defined by

$$
P_{i}\left(\sigma_{i}=s \mid \eta\right)=\frac{1}{2}\left(1+s \tanh \left(\beta \sum_{j \in \mathbb{Z}^{d}} k(i-j) \eta_{j}\right)\right)
$$

for an interaction function $k$ of finite range, non-negative, but not necessarily symmetric (cf. quasireversible PCA in [24]).

Under condition (4.2), we have, for all probabilities $\mu$,

$$
\left(P^{n} \mu\right) \circ T^{-1}=P^{n}\left(\mu \circ T^{-1}\right),
$$

from which the equality $\nu^{+}=\nu^{-} \circ T^{-1}$ follows.

Consider, now, the transition probability $P \circ T^{-1}$. This is the transition probability of a decreasing (repulsive) PCA which satisfies: $\left(P \circ T^{-1}\right) \nu^{+}=\nu^{-}$and $\left(P \circ T^{-1}\right) \nu^{-}=\nu^{+}$. If $\nu^{+} \neq \nu^{-}$(in the above example, it would correspond to a phase transition of associated Gibbs measures), then the measure $\alpha \nu^{+}+(1-\alpha) \nu^{-}$is stationary for the PCA $P \circ T^{-1}$ if and only if $\alpha=1 / 2$, while for other values of $\alpha$ this measure is periodic with period two.

This remark allows to produce an infinity of measures which are stationary for $P$ but not for the repulsive PCA $P \circ T^{-1}$.

P.-Y. Louis thanks, for their kind hospitality, the Mathematics' Department of Padova University and the Interacting Random Systems group of Weierstrass Institute for Applied Analysis and Stochastics in Berlin, where part of this work was done.

\section{REFERENCES}

[1] A.R. Barron, The strong ergodic theorem for densities: Generalized Shannon-McMillan-Breiman theorem. Ann. Probab. 13 (1985) 1292-1303.

[2] S. Bigelis, E.N.M. Cirillo, J.L. Lebowitz and E.R. Speer, Critical droplets in metastable states of probabilistic cellular automata. Phys. Rev. E 59 (1999) 3935-3941.

[3] P. Brémaud, Markov chains. Gibbs fields, Monte-Carlo simulation, and queues. Springer-Verlag, New York, Texts in Appl. Math. 31 (1999).

[4] P. Dai Pra, Ph.D. Thesis. Rutgers University (1992).

[5] D.A. Dawson, Synchronous and asynchronous reversible Markov systems. Canad. Math. Bull. 17 (1974/75) 633-649.

[6] H.-O. Georgii, Gibbs measures and phase transitions. Walter de Gruyter \& Co., Berlin, de Gruyter Stud. in Math. 9 (1988).

[7] S. Goldstein, R. Kuik, J.L. Lebowitz and C. Maes, From PCAs to equilibrium systems and back. Comm. Math. Phys. 125 (1989) 71-79.

[8] X. Guyon, Champs aléatoires sur un réseau. Modélisations, statistique et applications, Techniques stochastiques. Masson, Paris (1992).

[9] K. Handa, Entropy production per site in (nonreversible) spin-flip processes. J. Statist. Phys. 83 (1996) 555-571.

[10] R. Holley, Free energy in a Markovian model of a lattice spin system. Comm. Math. Phys. 23 (1971) 87-99.

[11] O. Kozlov and N. Vasilyev, Reversible Markov chains with local interaction, Multicomponent random systems. Dekker, New York, Adv. Probab. Related Topics 6 (1980) 451-469.

[12] H. Künsch, Nonreversible stationary measures for infinite interacting particle systems. Z. Wahrsch. Verw. Gebiete 66 (1984) 407-424.

[13] H. Künsch, Time reversal and stationary Gibbs measures. Stochastic Process. Appl. 17 (1984) 159-166.

[14] J.L. Lebowitz, C. Maes and E.R. Speer, Statistical mechanics of probabilistic cellular automata. J. Statist. Phys. 59 (1990) $117-170$.

[15] T.M. Liggett, Interacting particle systems, Vol. 276. Springer-Verlag, New York-Berlin (1985).

[16] F.J. Lopez and G. Sanz, Stochastic comparisons for general probabilistic cellular automata. Stat. Probab. Lett. 46 (2000) 401-410.

[17] C. Maes and S.B. Shlosman, Ergodicity of probabilistic cellular automata: A constructive criterion. Comm. Math. Phys. 135 (1991) 233-251.

[18] C. Maes and S.B. Shlosman, When is an interacting particle system ergodic? Comm. Math. Phys. 151 (1993) $447-466$. 
[19] C. Maes and K. Vande Velde, The (non-) Gibbsian nature of states invariant under stochastic transformations. Physica A 206 (1994) 587-603.

[20] V.A. Malyshev and R.A. Minlos, Gibbs random fields, Cluster expansions. Kluwer Academic Publishers, Dordrecht, Math. Appl. 44 (1991).

[21] F. Martinelli, Lectures on Glauber dynamics for discrete spin models, in Lectures on probability theory and statistics, SaintFlour (1997) 93-191. Springer, Berlin, Lecture Notes in Math. 1717 (1999).

[22] C. Preston, Random fields. Springer-Verlag, Berlin-New York, Lecture Notes in Math. 534 (1976).

[23] A.L. Toom, N.B. Vasilyev, O.N. Stavskaya, L.G. Mityushin, G.L. Kurdyumov and S.A. Pirogov, Discrete local Markov systems, in Stochastic Cellular Systems: Ergodicity, memory, morphogenesis, edited by R.L. Dobrushin, V.I. Kryukov and A.L. Toom. Manchester University Press, Manchester (1990) 1-182.

[24] N.B. Vasilyev, Bernoulli and Markov stationary measures in discrete local interactions, Locally interacting systems and their applications in biology. Pushchino (1976), edited by R.L. Dobrushin, V.I. Kryukov and A.L. Toom. Springer, Berlin, Lecture Notes in Math. 653 (1978). 